EPJ Web of Conferences 41, 02015 (2013)

DOI: $10.1051 /$ epjconf/20134102015

(C) Owned by the authors, published by EDP Sciences, 2013

\title{
Visible and ultraviolet photoelectron spectroscopy of fullerenes using femtosecond laser pulses
}

\author{
J. O. Johansson ${ }^{1}$, G. G. Henderson ${ }^{1}$, and E. E. B. Campbell ${ }^{1}$ \\ ${ }^{1}$ EaStCHEM, School of Chemistry, University of Edinburgh, West Mains Road, EH9 3JJ, UK
}

\begin{abstract}
Photoelectron spectra are presented for $\mathrm{C}_{60}$ excited with fs pulses of wavelengths 532 and $267 \mathrm{~nm}$. The spectra indicate a quick redistribution of the excitation energy. Excitation of SAMO states is observed with $532 \mathrm{~nm}$ excitation, but due to the relatively large photon energy of the $267 \mathrm{~nm}$ pulses, these orbitals are not populated for this wavelength.
\end{abstract}

\section{Introduction}

Fullerenes are often used as model systems for studying and attempting to understand the dynamical behaviour of relatively large organic molecules and nanoparticles. In comparison to more complicated molecules of comparable size, the high symmetry and the fact that fullerenes only consist of one element, means that theoretical modelling is simplified. Since fullerenes may also find applications in molecular scale electronics and are important constituents of organic solar cells, information about their electronic structure is important. We have recently shown that it is possible to study the so-called superatom molecular orbitals (SAMOs) of gas-phase fullerenes, using a combination of fs laser ionisation and angular-resolved photoelectron spectroscopy [1]. The SAMOs are diffuse, hydrogen-like molecular orbitals that form nearly-free electron bands in solids, and are therefore of practical interest [2]. In this contribution, we present photoelectron spectra (PES) of fullerenes obtained using ultraviolet and visible laser pulses of ca. 100 fs duration.

\section{Experimental setup}

Gas-phase $\mathrm{C}_{60}$ was introduced into a vacuum chamber by subliming purified $\mathrm{C}_{60}$ at a temperature of $500{ }^{\circ} \mathrm{C}$ in a resistively heated oven. To generate fs pulses with wavelength $267 \mathrm{~nm}$, the $800 \mathrm{~nm}, 120$ fs output of a Ti:Sapph regenerative amplifier was frequency doubled in a BBO crystal. The polarisation of the residual $800 \mathrm{~nm}$ beam was rotated to match that of the second harmonic and the relative delay between the two beams was controlled by a calcite plate. The two beams were then passed through another BBO crystal to produce the third harmonic with a wavelength of $267 \mathrm{~nm}$. The pulse duration was estimated to be similar to that of the fundamental beam. In addition to the third harmonic used for the experiments, a non-collinear parametric amplifier (NOPA) was used to produce pulses with a wavelength of $532 \mathrm{~nm}$ and pulse duration of $94 \mathrm{fs}$. This wavelength was chosen to match that of the second harmonic of a Nd:YAG laser, which was also used (with 4 ns pulse duration).

The laser beam intersected the effusive molecular beam at right angles and photoelectrons were extracted using a similar set of electrodes to those presented in reference [3]. The electrons were

This is an Open Access article distributed under the terms of the Creative Commons Attribution License 2.0, which permits unrestricted use, distribution, and reproduction in any medium, provided the original work is properly cited. 
projected onto a combination of microchannel plates and a phosphor screen and the image on the screen was recorded using a CCD camera. The electrode configuration allows for velocity mapping, resulting in the simultaneous recording of kinetic and angular photoelectron distributions. Typically $10^{6}-10^{7}$ laser shots were used to produce one "velocity map image" (VMI). The recorded 2D distributions were inverted to recover the initial 3D distribution of emitted photoelectrons, using a modified version of POP [4] which included up to the $10^{\text {th }}$ Legendre polynomial.

\section{Results and discussion}

When exciting fullerenes with ns laser pulses with photon energy below the ionisation threshold, quick energy redistribution leads to vibrational excitation of the molecules. Since the dissociation energy of fullerenes is larger than the ionisation energy, the molecules can be heated to such an extent that the vibrational energy content is enough to ionise the molecules [5]. This process is similar to thermionic emission in solids. The resulting PES is only comprised of thermally emitted electrons and no structure is seen, so that no information about the discrete electronic energy levels can be obtained. In the fs case, on the other hand, the excitation energy does not have time to couple to vibrations, and excited electronic states can be observed. As an example, this is shown in Figure 1, where a comparison between $\mathrm{ns}$ and fs excitation is shown for $532 \mathrm{~nm}$ excitation. The isotropic electron emission seen in the VMI image in Figure 1 (a) is due to the statistical nature of the electron emission, where no memory of the laser polarisation is preserved. In contrast, fs excitation results in some structure superimposed on a thermal background. The two low-kinetic energy peaks in the fs spectrum shown in Figure 1 (b) have been identified as the s- and p-SAMOs [1].
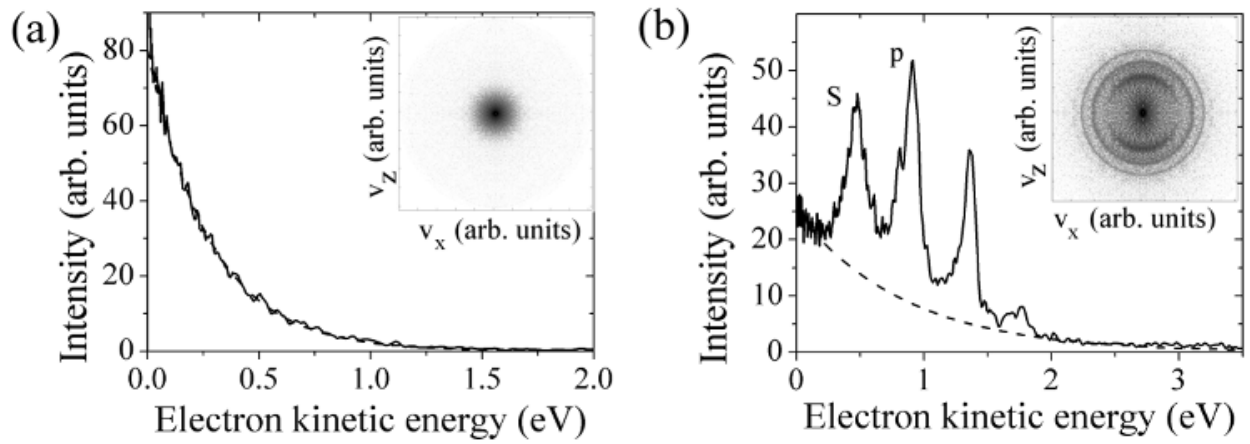

Fig. 1. PES obtained using $532 \mathrm{~nm}$ excitation for (a) ns pulses of $5 \times 10^{10} \mathrm{Wcm}^{-2}$ intensity and (b) $94 \mathrm{fs}$ pulses of intensity $2 \times 10^{12} \mathrm{Wcm}^{-2}$. The PES are obtained from the VMI images shown in the insets (where the laser polarisation was parallel with the $v_{z}$ axis). The dashed lines are fitted thermal electron distributions. As can be seen in (a), ns excitation results in a purely statistical electron emission whereas in (b), a discrete structure can be seen superimposed on the thermal background. The peaks labelled s and $\mathrm{p}$ were tentatively assigned as the s- and p-SAMOs in reference [1].

The results obtained using $267 \mathrm{~nm}$ are presented in Figure 2 (a). There is no strong indication of electrons with a thermal energy distribution in this case. Wurz and Lykke showed that exciting with the same wavelength using ns pulses, results in predominantly thermal electron emission [6]. In the results presented here, using fs pulses, the signal has a cut-off at a kinetic energy corresponding to a direct two-photon ionisation process, in contrast to the ns experiments reported in the literature. The largest part of the signal appears at lower kinetic energies, indicating that after the first absorbed photon, the energy is redistributed to lower-lying electronic states. These states are subsequently one-photon ionised with the conservation of vibrational energy, as illustrated in Figure 2 (b). The lowest SAMO should be seen at a kinetic energy of $2.75 \mathrm{eV}$, however, there is no clear peak in this region. It could be possible to populate the SAMOs using a second photon from a lower lying state, populated through internal conversion, and a third photon to ionise this state (in a $1+1+1$ ionisation 
process). However, the results show that the $1+1$ photon signal dominates the signal, as expected. In contrast, when using $532 \mathrm{~nm}$, the SAMOs may be populated by the last exciting photon through internal conversion and subsequently ionised by one more photon in a $3+1$ ionisation process (Figure 2b).
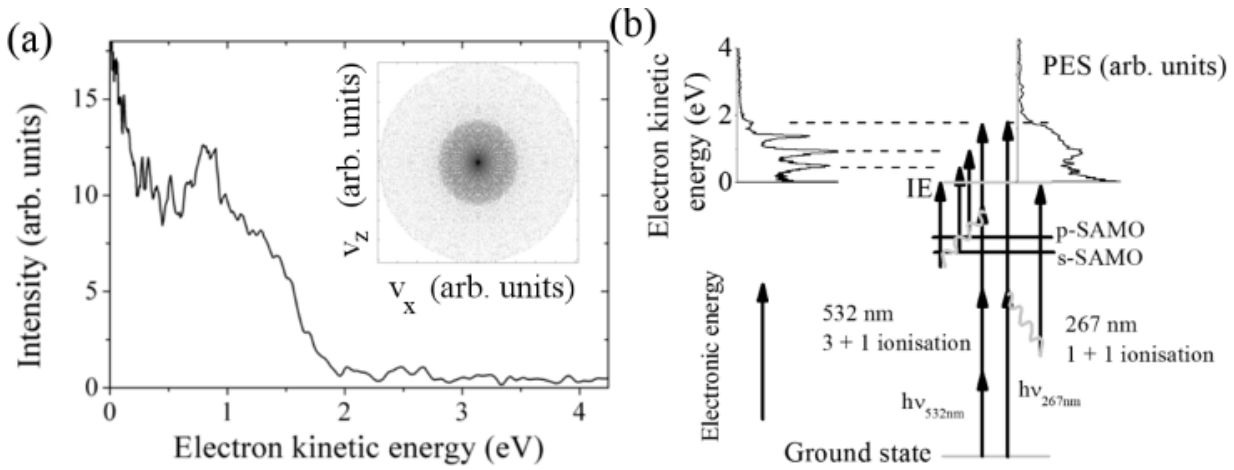

Fig. 2. (a) Angle-integrated PES of $\mathrm{C}_{60}$ obtained after $267 \mathrm{~nm}, 120 \mathrm{fs}$ laser excitation of intensity $2 \times 10^{11} \mathrm{Wcm}^{-2}$.

The inset shows the corresponding VMI. (b) Schematic figure showing a possible explanation of the experimental results. A direct photoexcitation takes place from the ground state to some initially excited state with one photon in the $267 \mathrm{~nm}$ case and three in the $532 \mathrm{~nm}$ case. After quick internal conversion to other electronic states, a manifold of different states can be accessed (as indicated by the grey wiggly lines). These states are subsequently one-photon ionised with the conservation of vibrational energy.

\section{Conclusion}

We have presented results from fs laser VMI photoelectron spectroscopy of $\mathrm{C}_{60}$ that indicate that, when using fs pulses of $267 \mathrm{~nm}$ wavelength, it is not possible to excite the s-SAMO using one photon, as expected. Since the second photon may directly ionise the molecule, other higher-order photon processes are not detected. In contrast, using $532 \mathrm{~nm}$, the SAMOs can be populated directly from the initial excited state through internal conversion and, once populated, be single-photon ionised.

\section{Acknowledgements}

The authors acknowledge EaStCHEM for funding. JOJ acknowledges the Edinburgh Fund for financial support for the nonlinear crystals.

\section{References}

1. J.O. Johansson, G.G. Henderson, F. Remacle, E.E.B. Campbell, Phys. Rev. Lett. 108, 173491 (2012)

2. M. Feng, J. Zhao, H. Petek, Science 320, 359 (2008)

3. M. Kjellberg, O. Johansson, F. Jonsson, A.V. Bulgakov, C. Bordas, E.E.B. Campbell, K. Hansen, Phys. Rev. A 81, 023202 (2010)

4. G.M. Roberts, J.L. Nixon, J. Lecointre, E. Wrede, J.R.R. Verlet, Rev. Sci. Intrum. 80, 53104 (2009)

5. E.E.B. Campbell, G. Ulmer, I.V. Hertel, Phys. Rev. Lett. 67, 1986 (1991)

6. P. Wurz, K.R. Lykke, J. Chem. Phys. 95, 7008 (1991) 